\title{
ANÁLISE DE GAME ENGINE PARA PROTOTIPAÇÃO DE OBJETO DE APRENDIZAGEM EM REALIDADE AUMENTADA
}

\section{GAME ENGINE ANALISYS FOR PROTOTIPATION OF A LEARNING OBJECT IN AUGMENTED REALITY}

\author{
Marta Cristina Goulart Braga, Dra. Eng. \\ Faculdade Energia de Administração e Negócios (FEAN) \\ e-mail: marta@goul.com.br
}

Palavras-chave: Objeto de aprendizagem, realidade aumentada, motor de jogo

\begin{abstract}
A análise de ambientes de desenvolvimento faz parte da etapa de planejamento de sistemas. Esta situação se aplica à prototipação de um Objeto de Aprendizagem (OA) em Realidade Aumentada (RA), pois é necessário verificar a viabilidade técnica do empreendimento. Neste caso, o ideal é que se tenha um ambiente adequado, que proporcione o gerenciamento de recursos, facilite o processo de desenvolvimento e o pipeline de renderização. Desta forma foi realizada uma análise de ambientes de desenvolvimento para RA, com o propósito de atender a certos requisitos técnicos e de licença de uso. A partir de análise prévia foi escolhido o OpenSpace3D para avaliação. Este ambiente demonstrou atender aos requisitos pretendidos superando as expectativas.
\end{abstract}

\section{Key-words: Learning Object, Augmented Reality, Game Engine}

The analysis of development environments is part of the sistem planning stage.This situation applies to the prototyping of an object of learning in augmented reality, because it is necessary to verify the technical feasibility of the project. In this case, the ideal is to have a proper environment, which enables the management of resources, facilitate the development process and the rendering pipeline.In this way it was carried out an analysis of development environments for $R A$, in order to meet certain technical and usage license requirements of the project. From prior analysis was chosen OpenSpace3D for evaluation. This environment has been shown to meet the desired requirements surpassing expectations.

\section{Introdução}

A análise de viabilidade é uma etapa do processo de desenvolvimento de software e este artigo trata da busca por possibilidades técnicas para $\mathrm{o}$ desenvolvimento de um Objeto de Aprendizagem (OA) em Realidade Aumentada (RA) para a Geometria Descritiva. O objetivo é encontrar um ambiente de software favorável e que atenda a determinados requisitos técnicos. Por se tratar da RA a tarefa não é trivial, pois dentre os sistemas existentes há muitos que estão defasados em relação à evolução dos sistemas operacionais, ou são comercializados exigindo grande despesa. É esperado identificar um Integrated Development Environment (IDE) que dê apoio à edição, estruturação de recursos e processo de renderização (pipeline), bem como deve enquadrar-se como open-source. 


\section{$16^{\circ}$ \\ ERGODESIGN USIHC CINAHPA}

Anteriormente foi realizada uma pesquisa de doutorado que resultou em um protótipo de baixa fidelidade de um OA para a aprendizagem de Geometria Descritiva, momento em que foi aplicado teste com usuários e geradas diretrizes de design de interação (BRAGA, 2012). No momento presente é necessário evoluir para um protótipo de média fidelidade, visando um processo cíclico de testes com usuário para aprimoramento. Este artigo apresenta a avaliação da plataforma Openspace $3 D$ para desenvolvimento de games que foi escolhida, após uma pesquisa prévia, dentre as IDE de RA e de game, aquelas disponíveis no mercado. Esperase, a partir da avaliação, determinar se a plataforma é adequada para a realização do protótipo de média fidelidade.

$\mathrm{O}$ artigo apresenta o conceito de OA, características da RA, tipos de interação, processo de funcionamento, dispositivos e software, uma análise do ambiente Openspace $3 D$, a partir de certos requisitos técnicos e de licença de uso e conclusão com apresentação de sugestões para futuros trabalhos.

\section{Referencial teórico}

\subsection{Objetos de Aprendizagem}

Há uma série de definições para Objetos de Aprendizagem, mas não tem sido simples definir o que são e como diferenciá-los de outros tipos de materiais de aprendizagem. $\mathrm{O}$ fato se deve à grande variedade nas características destes objetos: tamanho, escopo, conteúdo, design e implementação técnica, que são essencialmente os tópicos para a definição do conceito. Há um detalhe em comum entre as diversas definições que é a forma como eles são criados, utilizados e armazenados em detrimento do seu aspecto estético (SMITH, 2004). Dentre as variadas definições a apresentada pelo Learning Technology Standards Committee (LTSC) do IEEE torna o conceito bastante abrangente:

"Objeto de Aprendizagem é definido aqui como qualquer entidade, digital ou não digital, que pode ser utilizada, reutilizada ou referenciada na aprendizagem suportada pela tecnologia. Exemplos de tecnologia de suporte de aprendizagem incluem: $16^{\circ}$ Ergodesign - Congresso Internacional de Ergonomia e Usabilidade de Interfaces Humano Tecnológica: Produto, Informações Ambientes Construídos e Transporte

$16^{\circ}$ USIHC - Congresso Internacional de Ergonomia e Usabilidade de Interfaces Humano Computador

CINAHPA | 2017 - Congresso Internacional de Ambientes Hipermídia para Aprendizagem. sistemas de treinamento baseado em computadores, ambientes interativos de aprendizagem, sistemas inteligentes de instrução assistida por computador, sistemas de aprendizagem à distância, e ambientes de aprendizagem colaborativa. Exemplos de Objetos de Aprendizagem incluem: conteúdo multimídia, conteúdos instrucionais, objetivos de aprendizagem, softwares instrucionais, ferramentas de software, pessoas, organizações, ou eventos referenciados durante a aprendizagem suportada pela tecnologia" (IEEE, 2017, tradução da autora).

A produção de OA pode acontecer a partir de diversos tipos de tecnologia, tais como: Realidade Virtual, Flash, Realidade Aumentada ou Java.

\subsection{Realidade Aumentada}

A RA é uma interface híbrida composta pelo real sobreposto por entidades digitais. As exibições podem acontecer por diversos tipos de dispositivos, tais como monitores, projeções ou óculos especiais. Na RA, as interações ocorrem de forma diferenciada das interfaces gráficas convencionais (GUI-Graphical User Interfaces), que se utilizam do mouse e do teclado. Neste caso uma webcam rastreia um sinal manipulado pelas mãos do usuário, há ainda casos em que as próprias mãos fazem às vezes do sinal. A interface, cuja interação é tátil, é chamada de interface tangível (TUI - Tangible User Interfaces). De acordo com Shaer e Hornecker (2010) a interface tangível demonstra potencialidade para melhorar as interações dos usuários com as informações digitais. Os autores enfatizam que os estudos sobre interfaces tangíveis ainda estão embrionários. Por esse motivo é necessária uma extensa pesquisa para compreender plenamente suas implicações e, assim, se poderá utilizá-la para desenvolver outras tecnologias, que envolvam ainda mais o digital e o físico, e para orientar a concepção de interfaces tangíveis.

A RA continua em transformação e a tendência, além do uso de dispositivos móveis (p. ex.: para aplicações na educação, serviços e comércio), são os grandes formatos de displays para uso interno e externo (p. ex.: para serem usados em ações de 


\section{$16^{\circ}$ \\ ERGODESIGN USIHC CINAHPA}

$16^{\circ}$ Ergodesign - Congresso Internacional de Ergonomia e Usabilidade de Interfaces Humano Tecnológica: Produto, Informações Ambientes Construídos e Transporte

$16^{\circ}$ USIHC - Congresso Internacional de Ergonomia e Usabilidade de Interfaces Humano Computador

CINAHPA | 2017 - Congresso Internacional de Ambientes Hipermídia para Aprendizagem. marketing), uso de projetores (uma variação da RA chamada Realidade Aumentada Espacial ${ }^{1}$ ) ou de óculos-displays com câmeras acopladas.

Para Tori (2010, p. 160) “A RA espacial, ao liberar o usuário do uso de equipamentos sobre o próprio corpo e ao viabilizar experiências coletivas, abre inúmeras possibilidades de aplicação, tanto em artes e entretenimento, como em educação, e como ferramenta de produtividade". O autor aponta os projetores (mesmo os de mão e até os embutidos em celulares) como uma tendência na RA.

Outras variações da RA já se encontram em andamento: a Hiper-Realidade, que incorpora objetos virtuais inteligentes ao mundo real e o Cross-reality, que usa sensores espalhados no ambiente real para integrar informações do mundo online ao real (LANDAY; PARADISO, 2009, LIFTON et al., 2009). Mas, o futuro da RA, como diz Carmigniani e Furht (2011), ainda está na infância.

A evolução da RA e o surgimento de novos dispositivos determinam as forma de interação com esta tecnologia e impactam na escolha de um ambiente de desenvolvimento, pois o IDE precisa dar suporte a diversos dispositivos.

\subsubsection{Tipos de interação do usuário com a RA}

Interface é aquilo que permite ao homem interagir com dispositivos digitais. É o que faz a intermediação da comunicação humana com microprocessadores. Em outras palavras, é o que torna as funcionalidades de produtos visíveis, acessíveis e usáveis. O design de interface é o projeto da representação onde ocorrem as experimentações de interação. É aquilo que se vê, ouve e sente. $O$ design de interface faz parte do design de interação e interação tem a ver com o comportamento que permite que haja troca de informação entre entidades (pessoas, máquinas e sistemas) e por diversas maneiras. Design de interação corresponde a projetar para as experiências de troca, com foco nas atitudes das

\footnotetext{
${ }^{1}$ A Realidade Aumentada Espacial é aquela em que a exibição é feita por um projetor sobre objetos físicos, por exemplo: uma parede (BIMBER; RASKAR, 2004).
}

pessoas (SAFFER, 2010).

O design de interação surgiu com a conscientização dos profissionais da engenharia de hardware de que os primeiros computadores comercializados, para o público em geral, não eram máquinas fáceis de entender e usar. Eles perceberam a necessidade de compreender o comportamento dos usuários, de como eles se comunicam e interagem. Esse foi o caminho escolhido para projetar a diversidade de dispositivos interativos e variedade de mídias que se multiplicaram com a evolução das Tecnologias de Informação e Comunicação (TIC).

Broll et al (2005), propõem a seguinte classificação para os tipos de interação em RA: interação espacial, interação baseada em comandos, interação de controle virtual e interação de controle físico.

Interação espacial: se caracteriza pela interação espacial da manipulação das propriedades espaciais dos objetos físicos. Por exemplo: apontando ou fazendo gestos com as mãos e com os dedos com um dispositivo acoplado a mão com a qual é possível apontar e fazer gestos dinâmicos para manipular objetos virtuais.

Interação baseada em comandos: comandos por gesto, postura, movimento dos olhos e ou comando por voz para a execução de determinadas funcionalidades. Um exemplo deste tipo de interação são gestos e posturas sem quaisquer dispositivos, simplesmente objetos virtuais são manipulados com as mãos. Câmeras 2D podem ser utilizadas, mas no caso da Figura 1 é usada uma câmera 3D que consegue localizar a posição 3D dos usuários e objetos do virtual.



Figura 1: Interação baseada em comando. Fonte: (BRAGA, 2012). 


\section{$16^{\circ}$ \\ ERGODESIGN USIHC CINAHPA}

Interações de controle virtual: são baseadas em metáforas que atendem a certas funcionalidades, por exemplo, um menu elaborado por ferramentas 3D (Widgets 3D) (Figura 2).
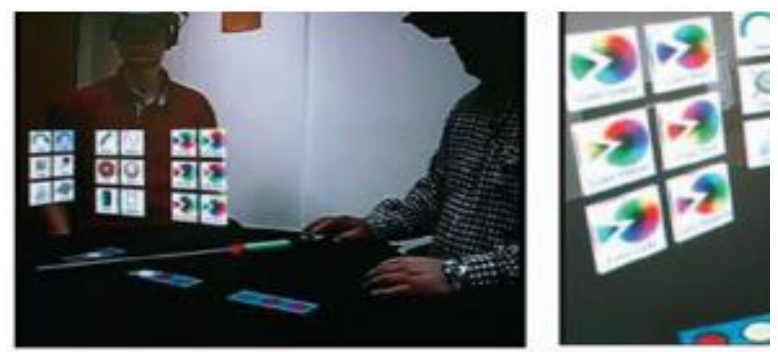

Figura 2: Interação de controle virtual. Fonte: (BROLL et al., 2005).

Interações de controle físico: são baseadas em objetos físicos que podem controlar tanto objetos físicos quanto virtuais (BROLL et al., 2005). Nesta categoria, incluem-se os controles remotos, como p. ex.: o Wii.

\subsubsection{Processo básico de funcionamento da RA}

Uma das possibilidades de configuração de hardware para executar um software de RA se compõe de um computador ${ }^{2}$ uma câmera e um display. A função da câmera é rastrear um sinal ${ }^{3} \mathrm{e}$ seu movimento, comumente um marcador (Figura 3) manipulado pelo usuário, para que a sua posição seja identificada e para que essa informação seja enviada continuamente para processamento. Há casos em que as próprias mãos são rastreadas, nesta situação a mão é o sinal.

\footnotetext{
${ }^{2} \mathrm{O}$ termo computador usado aqui compreende qualquer dispositivo, tais como: computador desktop, notebook ou dispositivos móveis com unidade de processamento, placas de vídeo e som e demais componentes necessários ao funcionamento completo do aparelho. ${ }^{3}$ Atualmente é possível que o sinal seja qualquer objeto físico: uma figura impressa, luva especial, uma lata de refrigerante, ou mesmo as mãos. Devido ao fato de neste trabalho ter se utilizado o termo "marcador" no desenvolvimento do protótipo, daqui para frente qualquer tipo de "sinal" será referenciado como "marcador" (nota da autora).
}

$16^{\circ}$ Ergodesign - Congresso Internacional de Ergonomia e Usabilidade de Interfaces Humano Tecnológica: Produto, Informações Ambientes Construídos e Transporte

$16^{\circ}$ USIHC - Congresso Internacional de Ergonomia e Usabilidade de Interfaces Humano Computador

CINAHPA | 2017 - Congresso Internacional de Ambientes Hipermídia para Aprendizagem.

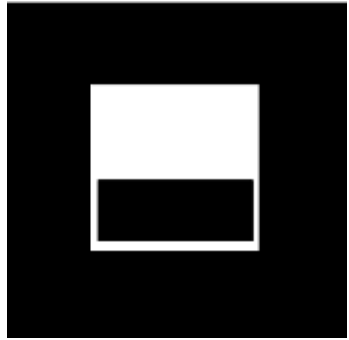

Figura 3: Marcador. Fonte: Acervo da autora.

A Unidade Central de Processamento (Central Processing Unit (CPU) identifica o marcador e procura pelo comando a ser executado ou por uma image $^{4}$ armazenada no disco rígido, que lhe tenha sido atribuída, com o objetivo de ser enviada ao display, para exibição conjunta com a imagem do marcador e tudo o mais que estiver sendo capturado pela câmera (BIMBER; RASKAR, 2004, FUA; LEPETIT, 2007). Esta é uma forma simplificada de explicar o fluxo do processo (Figura 4), contudo, cada etapa tem métodos e técnicas específicas, p. ex.: métodos de rastreamento, captação de imagem, calibragem de câmera, exibição, rendering, cálculos dos sistemas de coordenadas 3D, para que se alinhem aos registros de coordenadas $2 \mathrm{D}$.

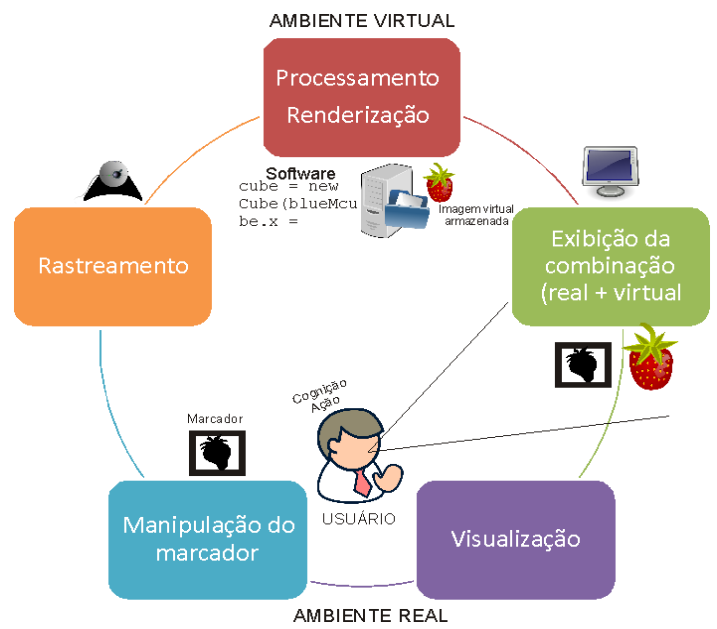

Figura 4: Configuração e fluxo do processo da RA. Adaptado de Fua e Lepetit (2007).

Fonte: Diagrama produzido pela autora. Ilustrações de Open Clip Art Library.

A seguir são apresentados alguns equipamentos, os

\footnotetext{
${ }^{4}$ Imagem que pode ser em 3D estática ou animação, uma imagem fotográfica ou um vídeo (nota da autora).
}

Realização:

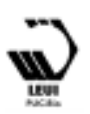


$16^{\circ}$ Ergodesign - Congresso Internacional de Ergonomia e Usabilidade de Interfaces Humano Tecnológica: Produto, Informações Ambientes Construídos e Transporte

$16^{\circ}$ USIHC - Congresso Internacional de Ergonomia e Usabilidade de Interfaces Humano Computador

CINAHPA | 2017 - Congresso Internacional de Ambientes Hipermídia para Aprendizagem. mais comuns.

\subsubsection{Hardware}

\section{Displays}

Os displays são sistemas de formação de imagem que utilizam um conjunto de componentes ópticos, eletrônicos e mecânicos para gerar imagens em algum ponto entre os olhos do observador e o objeto físico a ser aumentado. Dependendo do sistema utilizado, a imagem pode ser exibida em uma superfície plana ou mesmo em superfícies não planas (BIMBER; RASKAR, 2004).

As formas de exibição foram divididas em três tipos: head worn, handheld e projective.

Head worn: dispositivo montado sobre a cabeça $\mathrm{e}$ que se apresenta de duas formas: optical seethrough e Video see-through.

Optical seethrough: capacete ou óculos com lente, que consiste da sobreposição da RA em lentes transparente.

Video seethrough: que utiliza captura de vídeo por câmeras com um fundo para sobreposição e display opaco.

Handheld display: display de LCD manual com câmera incorporada. Uma espécie de monitor de mão. Os dispositivos móveis, como os celulares estão nesta categoria.

Projective. Uso de projetores com projeção direta sobre objetos físicos que dispensa o uso de óculos ou monitores (AZUMA et al., 2001). Também chamada de Realidade Aumentada Espacial (Spatial Augmented Reality (SAR).

\section{Outros dispositivos}

Sistema de luvas em lycra colorida, para rastreamento por webcam. O desenvolvimento é do aluno de graduação Robert Wang do MIT's Computer Science and Artificial Intelligence Laboratory.
Mochila com RC200 com dispositivos de sobreposição de camadas, luvas e marcadores coloridos e hardware de rastreamento óptico.

A Iris Tablet desenvolvida pelos designers chineses Liu Wei, Yao Kai Chi, Hong Ruei Hong e Cheng Ya Fang é um novo conceito que combina um touchpad transparente com um display OLED ${ }^{5}$ com capacidades para RA (YANKO DESIGN, 2012).

\subsubsection{Software}

Há diversos sistemas disponíveis para desenvolver projetos de RA, no entanto, uma grande maioria encontra-se estagnada, não tendo acompanhado a evolução dos sistemas operacionais, hardware e dispositivos. Muitas vezes são pesquisas de mestrado ou doutorado que dão lugar a novos projetos. É o caso, por exemplo, do Studierstube. As pesquisas iniciais do Studierstube foram realizadas na Universidade Tecnológica de Viena, de 1996 a 2004. Em 2005, o projeto mudou para o Institute for Computer Graphics and Vision, Graz University of Technology. Graz, Áustria. Studierstube roda em Windows XP e está disponível gratuitamente para baixar sob GPL. A versão para celular não é disponibilizada ao público. Studierstube permite a colaboração face a face e remota (SANTIN; KIRNER, 2008, STUDIERSTUBE, 2012).

O Artoolkit é um Software development Kit (SDK) que sobreviveu ao tempo. Originalmente foi desenvolvida pelo Dr. Hirokazu Kato da Universidade de Osaka, Universidade da cidade de Hiroshima e do Interactive Media Design Laboratory - Graduate School of Information Science - Nara Institute of Science and Technology. Liberada para uso em 1999 é gratuito e hoje encontra-se na versão 5.3.2. Essa biblioteca foi largamente utilizada, tanto naqueles sistemas que ficaram parados no tempo, quanto continua rodando hoje em outros. É o caso, por exemplo da ferramenta de autoria BuildAR Pro do HIT Lab NZ que continua sendo desenvolvido e comercializado pela Envisage AR e roda no Windows 10. Já a

${ }^{5}$ OLED é a sigla em inglês de Organic Light-Emitting Diode (nota da autora).
Realização:

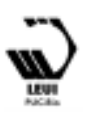




\section{$16^{\circ}$ \\ ERGODESIGN USIHC CINAHPA}

versão gratuita não foi atualizada desde 2008.

Existem extensões adaptadas a ambientes de desenvolvimento 3D, por exemplo: o Sketchup, Maya, dentre outros. Destaca-se o plugin da Armedia SDK que possui uma versão de experimentação com limites funcionais e a versão paga sem limitações. Algumas plataformas de games, tais como Unity $3 D$, Unreal Engine e Openspace $3 D$ possuem extensões ArtoolKit.

Vuforia SDK é outra biblioteca que permite desenvolver RA para diversas plataformas, incluindo aplicativos móveis. Possui versão para Android, IOS, Windows e plugin para Unity3D. Diferente da ArtoolKit, que é open source, Vuforia é comercializada. É possível baixar uma versão sem limite de funcionalidades, mas apenas para um projeto piloto, que caso venha a ser comercializado, a licença adequada deve ser adquirida $^{6}$.

\section{Avaliação de ambiente de desenvolvimento para protótipo de média fidelidade de OA em RA.}

Esta pesquisa teve início em tese de doutorado (BRAGA, 2012), naquela ocasião foi desenvolvido um protótipo de um OA de baixa fidelidade (Figura 5), que foi submetido a teste com usuário e gerou diretrizes, para o design de interação de OA em RA.

O primeiro protótipo de baixa fidelidade foi desenvolvido com o FlartoolKit uma biblioteca que permite o uso de navegador de internet e para alterar o action script 3, foi utilizada a versão acadêmica e gratuita do Flash Builder ${ }^{7}$, além do Eclipse $^{8}$ e Flex framework ${ }^{9}$. Estes dois últimos são open source.

\footnotetext{
${ }^{6}$ Uma lista de softwares de Realidade Aumentada encontra-se em: https://en.wikipedia.org/wiki/List_of_augmented_reality_software. ${ }^{7} \mathrm{O}$ Flash Builder está disponível em: http://www.adobe.com/br/products/flash-builder.html. ${ }^{8} \mathrm{O}$ Eclipse está disponível em: http://www.eclipse.org/.

${ }^{9} \mathrm{O}$ Flex framework está disponível em:

http://www.adobe.com/products/flex.html.
}

$16^{\circ}$ Ergodesign - Congresso Internacional de Ergonomia e Usabilidade de Interfaces Humano Tecnológica: Produto, Informações Ambientes Construídos e Transporte

$16^{\circ}$ USIHC - Congresso Internacional de Ergonomia e Usabilidade de Interfaces Humano Computador

CINAHPA | 2017 - Congresso Internacional de Ambientes Hipermídia para Aprendizagem.

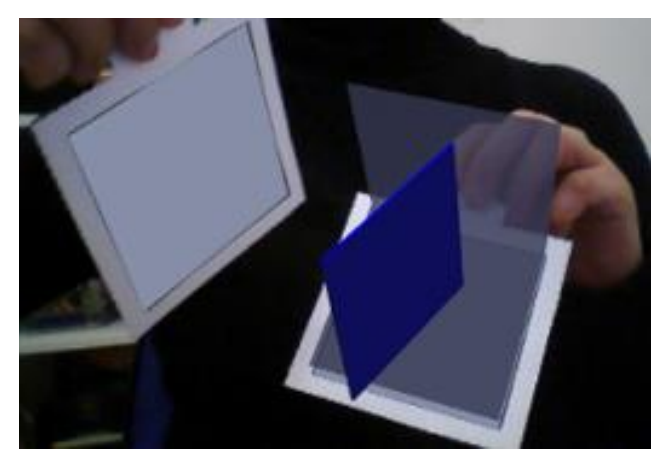

Figura 5: protótipo de baixa fidelidade. Fonte: acervo da autora.

Estes sistemas de desenvolvimento trazem um editor de linguagem de programação e o SDK, mas não possuem uma $3 D$ view possível de apresentar cenas e elementos modelados em 3D. Além destes aspectos, as funções e qualquer conexão necessária com dispositivos são realizadas por meio de linguagem de programação.

Quatro marcadores em preto e branco foram produzidos com pictogramas, metáforas dos elementos virtuais: diedro, plano, retas e uma casa Figura 6.

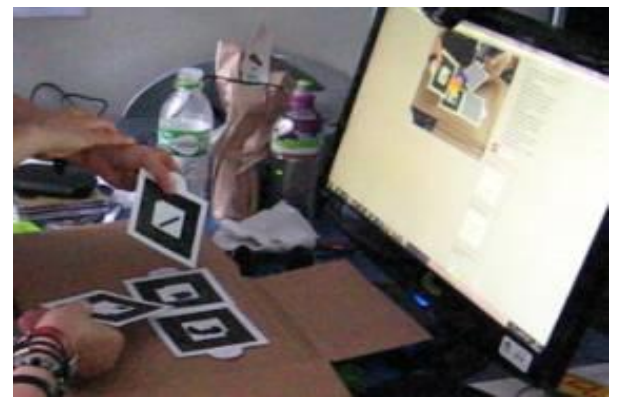

Figura 6: Teste com usuário.Ambiente não controlado. Font: acervo da autora.

\subsection{Requisitos}

Diante das expectativas atuais são os seguintes os requisitos para a escolha do ambiente de desenvolvimento do OA em RA:

O ambiente deve ser open source, deve apoiar a edição de cenas, estruturação de recursos, conexão com dispositivos de interação de comando e processo de renderização (pipeline) do OA. Precisa 


\section{$16^{\circ}$ \\ ERGODESIGN USIHC CINAHPA}

gerar um executável para ser distribuído devendo rodar em sistemas operacionais mais atuais: Windows, Linux, OSX, Android e IOS. Precisa gerar marcadores coloridos e deve, ainda, possibilitar que o OA seja apresentado em um navegador de internet.

\subsection{Resultado de pesquisa prévia}

A pesquisa prévia aconteceu por meio de visita aos sites de diversos sistemas, alguns foram baixados, instalado e executados para análise. Não foi possível identificar um ambiente de desenvolvimento de RA, ao mesmo tempo, open source e com versões atualizadas para rodar nos sistemas operacionais mais atuais. As opções existentes e com boas possibilidades são comercializadas. Por outro lado foi possível encontrar ambientes de games ${ }^{10}$ que possuem plugins para RA. É o caso da Unity3D, Unreal Engine e Openspace $3 D$. Especialmente Openspace $3 D$ é open source. Unity $3 D$ tem uma versão gratuita, com algumas restrições, para uso de entusiastas, apenas as versões pagas podem gerar produtos profissionais. Unreal Engine possui um plugin para RA comercializado.

Dentre esses ambientes chamou a atenção a Openspace $3 D$, por ser open source e possui plugin ArtoolKit. IDEs de games lidam com cenas e objetos 3D e estão preparadas para gerar executáveis para diversos sistemas operacionais atuais. Openspace $3 D$, além destas características, tem uma $I D E$ composta por um editor de cena simples e objetivo (Figura 7) e pelo $S D K$ que é o núcleo do sistema. $16^{\circ}$ Ergodesign - Congresso Internacional de Ergonomia e Usabilidade de Interfaces Humano Tecnológica: Produto, Informações Ambientes Construídos e Transporte

$16^{\circ}$ USIHC - Congresso Internacional de Ergonomia e Usabilidade de Interfaces Humano Computador

CINAHPA | 2017 - Congresso Internacional de Ambientes Hipermídia para Aprendizagem.

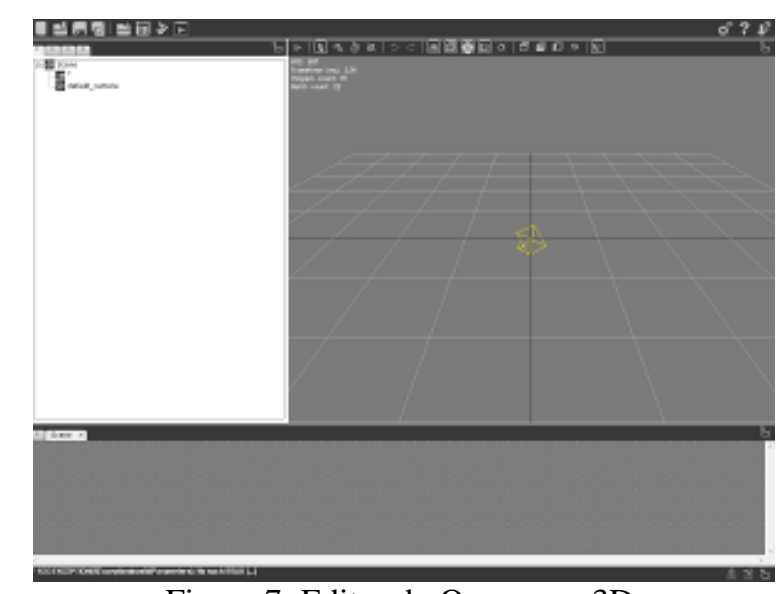

Figura 7: Editor do Openspace3D.

Fonte: Acervo da autora.

Através do editor é possível importar arquivos 3D, tais como: FBX, Colada, Obj, Blender, dentre outros. Há suporte para diversos dispositivos de interação para RA, por exemplo, óculos, sensor magnético, leap motion, sistemas de captura, rastreador de face. Pode enviar comandos para arduino e raspbarry PI. Pode exportar para navegador de internet e tem funções de comando para dispositivos.

Há funções para a geração de marcadores e até mesmo marcadores ilustrados coloridos para RA (Figura 8).

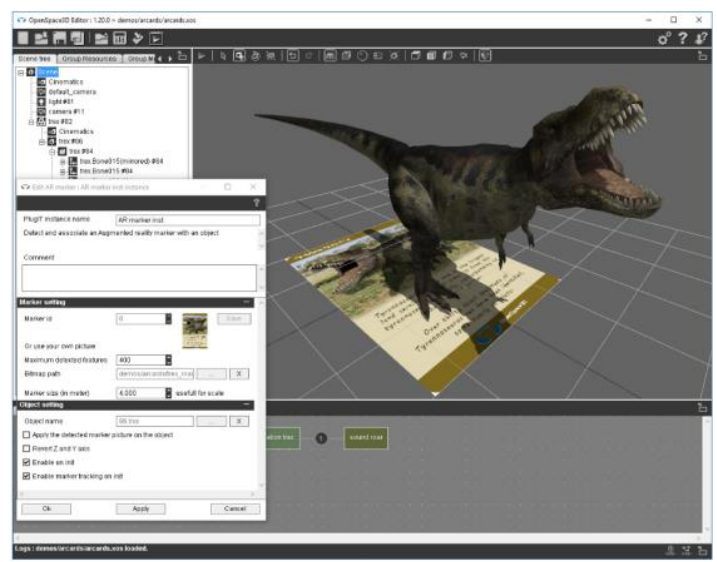

Figura 8: Geração de marcadores ilustrados coloridos. Fonte: OPENSPACE3D (2017).

Openspace $3 D$ apoia o gerenciamento de recursos, facilita o processo de desenvolvimento e o pipeline de renderização. Além destes aspectos, game

\footnotetext{
${ }^{10}$ Uma lista de game engine pode ser encontrada em: https://pt.wikipedia.org/wiki/Lista_de_motores_de_jogo.
} 


\section{$16^{\circ}$ \\ ERGODESIGN USIHC CINAHPA}

engines podem lidar com a física dos objetos e efeitos especiais (particles).

Há pontos fracos em Openspace $3 D$ e o principal problema encontra-se na importação de objetos gerados em programas 3D, por exemplo, a importação de arquivos nativos do programa Blender. No entanto há uma comunidade de desenvolvimento trabalhando para corrigir as falhas de Openspace3D.

As informações têm por base o conteúdo do site do ambiente e a confirmação foi feita através de instalação, execução e testes. Por suas características OpenSpace $3 D$ pode vir a viabilizar o projeto.

\section{Conclusão e futuros trabalhos}

O artigo apresentou uma análise do ambiente Openspace $3 D$, que é uma game engine open source. $\mathrm{O}$ objetivo foi de encontrar um sistema para o desenvolvimento de OA em RA a partir de certos requisitos, aqueles apresentados no item 3.1 Requisitos. Openspace $3 D$ superou as expectativas, pois apresenta funcionalidades que vão além dos requisitos esperados.

Openspace $3 D$ possui alguns defeitos de funcionamento, no entanto há uma comunidade de desenvolvedores corrigindo as falhas e novas versões continuam sendo ofertadas aos usuários.

Há grande possibilidade de Openspace $3 D$ vir a ser o ambiente para desenvolver uma nova versão do protótipo de OA de geometria descritiva, tanto a versão de média fidelidade, quanto de alta fidelidade e há fortes indícios que é o candidato a ser o ambiente de desenvolvimento do produto final.

Como futuros trabalhos sugere-se:

- Desenvolver novos marcadores coloridos.

- Criar novos objetos em 3D.

- Propiciar interações por comando para os objetos 3D.

- Criar menus de escolha, para adequar o OA as preferências de tela dos usuários. $16^{\circ}$ Ergodesign - Congresso Internacional de Ergonomia e Usabilidade de Interfaces Humano Tecnológica: Produto, Informações Ambientes Construídos e Transporte

$16^{\circ}$ USIHC - Congresso Internacional de Ergonomia e Usabilidade de Interfaces Humano Computador

CINAHPA | 2017 - Congresso Internacional de Ambientes Hipermídia para Aprendizagem.

\section{BIBLIOGRAFIA}

AZUMA, R. et al. Recent advances in augmented reality. IEEE Computer Graphics and

Applications, 2001, v. 21, n. 6, p. 34-

47.Disponível em:

http://ieeexplore.ieee.org/xpl/freeabs_all.jsp?arnum ber $=963459$. Acesso em: 8 jul. 2011.

BIMBER, O.; RASKAR, R. Spatial Augmented

Reality: merging real and virtual worlds,

Wellesley: A K Peters - Ltd,2004.

BRAGA, M.C.G. Diretrizes para o design de mídia em Realidade Aumentada: Situar a aprendizagem colaborativa online., 2012. Universidade Federal de Santa Catarina, 2012.

BROLL, W. et al. An infrastructure for realizing custom-tailored augmented reality user interfaces.

IEEE transactions on visualization and computer graphics, 2005 , v. 11 , n. 6 , p. $722-$ 33.Disponível em:

http://ieeexplore.ieee.org/xpl/freeabs_all.jsp?arnum ber $=1512022$. Acesso em: 9 abr. 2011.

CARMIGNIANI, J.; FURHT, B. Augmented Reality: An Overview. In 2011, Handbook of Augmented Reality. New York: Springer, 2011.

FUA, P.; LEPETIT, V. Vision Based 3D Tracking and Pose Estimation for Mixed Reality. In M. Haller, M. Billinghurst, B. H. Thomas, ORGS. 2007, Emerging technologies of augmented reality: interfaces and design. Idea Group Inc (IGI), 2007. p. 399.Disponível em:

http://books.google.com/books?id=WXHCyfEBx7 QC\&pgis=1. Acesso em: 16 abr. 2011.

IEEE. IEEE Learning Technology Standards Committee (LTSC).Disponível em: https://ieeesa.imeetcentral.com/ltsc/. Acesso em: 20 mar. 2017.

LANDAY, J.A.; PARADISO, J.A. Guest Editors' Introduction: Cross reality environments.Disponível em:

http://dspace.mit.edu/handle/1721.1/60069. Acesso em: 7 jan. 2012. 


\section{$16^{\circ}$ \\ ERGODESIGN USIHC CINAHPA}

$16^{\circ}$ Ergodesign - Congresso Internacional de Ergonomia e Usabilidade de Interfaces Humano Tecnológica: Produto, Informações Ambientes Construídos e Transporte

$16^{\circ}$ USIHC - Congresso Internacional de Ergonomia e Usabilidade de Interfaces Humano Computador

CINAHPA | 2017 - Congresso Internacional de Ambientes Hipermídia para Aprendizagem.

LIFTON, J. et al. Metaphor and Manifestation Cross-Reality with Ubiquitous Sensor/Actuator Networks. IEEE Pervasive Computing, IEEE Computer Society, 2009, v. 8, n. 3, p. 24-

33.Disponível em:

http://www.computer.org/portal/web/csdl//doi?doc= doi/10.1109/MPRV.2009.49. Acesso em: 7 jan. 2012.

OPENSPACE3D. Open Source Platform For 3D Environments.Disponível em:

http://www.openspace3d.com/. Acesso em: 20 mar. 2017.

SAFFER, D. Designing for Interaction: Creating Innovative Applications and Devices, EPub, New Riders, 2010.

SANTIN, R.; KIRNER, C. Sistema de Autoria em Ambiente Colaborativo com Realidade Aumentada, 2008. Universidade Metodista de Piracicaba, Piracicaba, 2008.

SHAER, O.; HORNECKER, E. Tangible User Interfaces, Now Publishers Inc,2010.Disponível em:

http://books.google.com/books?id=vh_tCfvK4M4 C\&pgis=1. Acesso em: 3 jan. 2012.

SMITH, R.S. Guidelines for Authors of: Learning Objects N. T. N. M. Consortium, ORG., McGraw-Hill Education,2004.Disponível em: http://archive.nmc.org/guidelines/. Acesso em: 3 maio 2011.

\section{STUDIERSTUBE. Augmented Reality - Institute for Computer Graphics and Vision.Disponível em: http://www.icg.tugraz.at/project/studierstube. Acesso em: 10 jan. 2012.}

TORI, R. Educação sem distância: as tecnologias interativas, São Paulo: Senac,2010.

YANKO DESIGN. Iris Tablet PC by Liu-Wei, Yao Kai-Chi, Hong Ruei-Hong \&amp; Cheng Ya-Fang» Yanko Design.Disponível em: http://www.yankodesign.com/2011/08/25/iris-mykinda-tablet/. Acesso em: 24 jan. 2012. 